\title{
Pegvisomant and Pasireotide LAR as second line therapy in acromegaly: clinical effectiveness and predictors of response
}

\author{
Sabrina Chiloiro 1,2 , Antonella Giampietro', Federica Mirra1, Federico Donfrancesco', Tommaso Tartaglione ${ }^{3,4}$, \\ Pier Paolo Mattogno5, Flavia Angelini ${ }^{2}$, Lauretti Liverana ${ }^{5}$, Marco Gessi ${ }^{6}$, Anile Carmelo5, Guido Rindi ${ }^{6,7}$, \\ Andrea Giustina ${ }^{8}$, Maria Fleseriu' ${ }^{9}$ Alfredo Pontecorvi ${ }^{1,2}$, Laura De Marinis ${ }^{1,2}$ and Antonio Bianchi ${ }^{1}$
}

1Pituitary Unit, Department of Endocrinology and Diabetes, Fondazione Policlinico Universitario A. Gemelli, IRCCS, Rome, Italy, 2Dipartimento di Medicina e Chirurgia Traslazionale, Università Cattolica del Sacro Cuore, Rome, Italy, ${ }^{3}$ Department of Radiology, Istituto Dermatopatico dell'Immacolata, IRCCS, Rome, Italy, ${ }^{4}$ Istituto di Radiologica, Università Cattolica del Sacro Cuore, Rome, Italy, ${ }^{5}$ Department of Neurosurgery, Fondazione Policlinico Universitario A. Gemelli, IRCCS, Università Cattolica del Sacro Cuore, Rome, Italy, ${ }^{6}$ Department di Pathology, Fondazione Policlinico Universitario A. Gemelli, IRCCS, Rome, Italy, ${ }^{7}$ Istitute of Pathology, Università Cattolica del Sacro Cuore, Rome, Italy, ${ }^{8}$ Endocrinology Vita-Salute San Raffaele University, IRCCS, San Raffaele Hospital, Milano, Italy, and ${ }^{9}$ Departments of Medicine (Endocrinology) and Neurological Surgery, and Pituitary Center, Oregon Health \& Science University, Portland, Oregon, USA

Correspondence should be addressed to L De Marinis

Email

Laura.Demarinis@unicatt.it

\begin{abstract}
Background: The treatment of acromegaly resistant to first-generation somatostatin receptor ligands (SRLs) is often difficult. Pegvisomant and Pasireotide LAR are mostly used in these subset of patients, as second line therapies. Choice of the type of second line therapies is difficult, since predictors of response are still unclear, impairing personalized therapy. We aimed to investigate predictors of response to Pegvisomant and Pasireotide LAR.

Methods: Seventy-four acromegaly patients entered this observational, cross-sectional and retrospective study if (i) resistant to high dose first-generation SRLs and (ii) treated with Pegvisomant and Pasireotide LAR for at least 12 consecutive months. Patients treated with radiotherapy in the previous 10 years were excluded.

Results: Fourty-one patients were treated with Pegvisomant and 33 with Pasireotide LAR. At the end of the study, acromegaly was controlled in 35 patients treated with Pegvisomant (85.4\%) and in 23 treated with Pasireotide LAR (69.7\%). In this cohort, a poor Pegvisomant response and a shorter progression free time were observed in cases with tumor extension to the third ventricle ( $P=0.004$, HR: 1.6, 95\% Cl: $1.2-4.6)$, with a Ki67-Li $>4 \%(P=0.004, \mathrm{HR}: 3.49$, $95 \% \mathrm{Cl}: 1.4-4.0)$ and with pre-treatment IGF-I $>3.3 \times \mathrm{ULN}(P=0.03$, HR: 1.3, 95\% CI: 1.1-6.0). A poor Pasireotide LAR response and a shorter progression free time were observed in cases with tumor extension to the third ventricle $(P=0.025, \mathrm{HR}: 1.695 \% \mathrm{Cl}: 1.4-3.4)$, pre-treatment IGF-I $>2.3 \times \mathrm{ULN}(P=0.049, \mathrm{HR}: 2.4,95 \% \mathrm{Cl}: 1.4-8.0)$, absent/low SST5 membranous expression ( $P=0.023 \mathrm{HR}: 4.5695 \% \mathrm{Cl}: 1.3-6.4)$ and in patients carried the d3-delated GHR isoform $(P=0.005$, HR: $11.37,95 \% \mathrm{Cl}: 1.3-20.0)$.
\end{abstract}

Conclusion: Molecular and clinical biomarkers can be useful in predicting the responsiveness to Pegvisomant and Pasireotide LAR.

European Journal of Endocrinology

(2021) 184, 217-229 


\section{Introduction}

Acromegaly is a chronic, systemic and complex disease. The main aims in the treatment of acromegaly should be oriented to the biochemical control of the hormonal hyper-secretion and to the management of the pituitary tumor (1). The mortality and morbidity rate of active acromegaly are higher than those of the general population, in relation to the increased risk of developing systemic complications (2).

Surgery is recommended as the primary treatment in the large majority of patients (1). The post-surgical remission rate ranges from 40 to $90 \%$ of cases, depending on tumor size and invasiveness and neurosurgeon expertize (3). The first-generation SRLs, Octreotide and Lanreotide, represent a cornerstone in the medical management of acromegaly. According to the most recent meta-analysis, first-generation somatostatin receptor ligands (first-generation SRLs) can induce the control of hormonal hypersecretion in approximately $55 \%$ of acromegaly patients (4). A not negligible group of patients required treatment with second-line and multimodal therapies, as GH receptor antagonist (Pegvisomant) and second generation SRLs (Pasireotide long-acting release). Although many predictive factors of responses to first-generation SRLs have been described (5), at this moment, clinical-biochemical and molecular predictors of responsiveness to Pegvisomant and Pasireotide LAR are not completely clarified. A personalized treatment may be useful for a prompt achievement of the therapeutic aims. In fact, both the last 2017 WHO classification of pituitary tumors and the latest guidelines on the management of aggressive acromegaly underline the importance of integrate clinical-biochemical and molecular data for achieving a tailored therapeutic approach $(6,7)$. Therefore, the aim of this study is the identification of potential biochemical and molecular biomarkers, able to predict the responsiveness to Pegvisomant and Pasireotide LAR, in acromegaly patients with resistance to first-generation SRLs.

\section{Subjects and methods}

\section{Subjects and protocol}

An observation, cohort and retrospective study (8) was performed on acromegaly patients, considered partially or completely resistant to first-generation SRLs. Treatment period ranged between June 2007 and December 2018. Data collection was performed until January 2020.
Patients were consecutively enrolled according to the following inclusion criteria:

(i) acromegaly patients with active disease, after neurosurgery and/or considered partial or complete resistant to first-generation SRLs, after at least 6 consecutive months of standard therapy and at least further 6 consecutive months of increased dose frequency (Lanreotide ATG 120 mg every 21 days or Octreotide LAR 30 mg every 21 days) (9);

(ii) patients on treatment with Pasireotide LAR or Pegvisomant in association to first-generation SRLs, for active acromegaly, for at least 12 consecutive months;

(iii) patients older than 18 years.

We excluded from the study patients who were pretreated with first generation SRLs before surgery, who underwent radiotherapy in the previous 10 years and those treated with Pasireotide LAR or Pegvisomant for less than 12 months. Baseline corresponded to the visit/day of first dose of Pegvisomant or Pasireotide LAR.

Active acromegaly was defined in cases with random GH higher than $1.0 \mathrm{ng} / \mathrm{mL}$ and IGF-I concentrations above the normal ranges for age (1). Partial response to first-generation SRLs was defined by a significant decrease ( $>50 \%$ ) of GH and/or IGF-I levels with no achievement of control and/or $>20 \%$ tumor shrinkage. Poor response or complete resistance to first-generation SRLs was defined by lack of significant decrease of GH and IGF-I levels and no tumor shrinkage or increase in tumor size (10).

The treatment choice for Pegvisomant or Pasireotide LAR was based on phsyician's clinical judgment in the absence of reference guidelines, which were first published in October 2018 (1).

During the follow-up, patients were biochemically evaluated at least every 6 months. Pegvisomant and Pasireotide LAR dosages were gradually increased during follow-up, until reaching biochemical acromegaly control or the maximum tolerate dose.

The end of the study corresponded to the last evaluation visit, when all patients underwent hormonal tests and pituitary MR.

\section{Outcome}

At the end of the study, patients were defined controlled if the IGF-I values were in the reference ranges for age and gender (at least in two consecutive measures) and random GH was below $1.0 \mathrm{ng} / \mathrm{mL}$ (1). Patients on treatment with 
Pegvisomant were evaluated only by serum IGF-I. IGF-I was expressed as IGF-I for upper limit of normality (ULN).

Gender, age (at acromegaly diagnosis and at study entry), GH and IGF-I × ULN values (at diagnosis, after pituitary surgery and after treatment with first-generation SRLs), tumor morphology (invasion of cavernous sinus, Knosp score, extension to third wentricle, tumor proliferative index (Ki-67), SST2 and SST5 expression, cytokeratin pattern and isoform of the GHR were investigated as potential predictors of the response to Pegvisomant plus first-generation SRLs or Pasireotide LAR.

\section{Knosp index}

Knosp index was evaluated by the same operator, though preoperative MR images, in according to Knosp classification (11). Grade 0 represented the normal condition of the CS space. The adenoma did not pass the tangent of the medial aspects of the supra- and intracavernous (internal carotid artery) ICA. Grade 1 was characterized by tumor extension that did not pass a line between the cross-sectional centers of the carotid arteries, the so -called 'intercarotid line'. Grade 2 was characterized by tumor extending beyond the intercarotid line, but not extending beyond or tangent to the lateral aspects of the intra - and supracavernous ICA. Grade 3 was characterized by tumor extending lateral to the lateral tangent of the intra- and supracavernous ICA. Grade 4 was characterized by total encasement of the intracavernous carotid artery) (11). Knosp grade 3 and grade 4 were considered cavernous sinus invasive adenoma (12). In addition, Knosp index (11) and invasion of the cavernous sinus and of the IIIrd ventricle were diagnosed through pituitary MRI and confirmed intra-operatory.

\section{Histopathological assessment}

Histopathological assessment was conducted according to our clinical practice $(12,13,14)$. All specimens were examined for pituitary hormones, Ki-67 antigen and somatostatin receptor (SST). The Ki-67 index was expressed as the maximum percentage of positive nuclei. SST expression was scored as previously proposed by Volante et al. (15), taking into account both the subcellular localization and the extent of the staining: score 0, no immunoreactivity; score 1, cytoplasmic immunoreactivity; score 2, membranous staining in $<50 \%$ of cells or incomplete membranous staining; score 3 , circumferential membranous staining in more than $50 \%$ of tumour cells. Expression of aip was scored semi- quantitatively (H-score), considering both the percentage of positive cells (0-100\%) and the staining intensity (0-3), total range 0-300 (16).

\section{GHR isoform assessment}

The GHR genotype (flfl, fld3, or d3d3) was determined from peripheral blood. At study entry, genomic DNA was extracted from $100 / 200 \mu / \mathrm{L}$ peripheral blood by Kit ILLUSTRA $^{\text {TM }}$ blood Genomic Prep Mini Spin GE Healthcare (for the rapid and reproducible isolation of high-quality genomic DNA from whole blood) for the detection of the GHR gene polymorphism. Polymorphisms were studied through PCR by Eppendorf® MasterMix (2.5×) (the 2.5× Eppendorf $®$ MasterMix contains Taq DNA polymerase

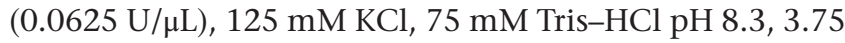
$\mathrm{mM} \mathrm{MgCl} 2,0.25 \%$ Nonidet ${ }^{\circledR}-\mathrm{P} 40,500 \mu \mathrm{M}$ of each dNTP and stabilizers. This corresponds to final concentrations in the PCR reaction of $1.25 \mathrm{UTaq}$ DNA polymerase, $50 \mathrm{mM} \mathrm{KCl}$, $30 \mathrm{mM}$ Tris- $\mathrm{HCl}, 1.5 \mathrm{mM} \mathrm{Mg}^{2+}$, and $200 \mu \mathrm{M}$ of each dNTP. Amplification was performed using primers, as described previously (17). 5'-TGTGCTGGTCTGTTGGTCTG-3', 5'-AGTCGGTTCCTGGGACGAGA-3', 5'-CCTGGATTAACA CTTTGCAGACTC-3', and as follows: 1 cycle at $94^{\circ} \mathrm{C}$ for 5 min (Denaturing); 35 cycles at $94^{\circ} \mathrm{C}$ for $30 \mathrm{~s}$ (Denaturing) $60^{\circ} \mathrm{C} 30 \mathrm{~s}$ (Annealing), $72^{\circ} \mathrm{C} 60 \mathrm{~s}$ (Extension); 1 cycle at $72^{\circ} \mathrm{C}$ for $7 \mathrm{~min}$ (last Extension). The amplification products were separated by electrophoresis on $2 \%$ agarose gel and visualized with the use of ethidium bromide. The full-length allele (GHRfl) is represented by a $935 \mathrm{bp}$ fragment and the exon 3-deleted allele (GHRd3) by a 532 bp fragment.

\section{Statistical analysis}

The primary objective of the study was the identification of biomarkers of responsiveness to treatment with Pegvisomant plus first-generation SRLs and Pasireotide LAR.

The patients cohort was described in its clinical and demographic features using descriptive statistics techniques. Normality of continuous variables was checked using Kolmogorov-Smirnov test. Quantitative variables were expressed as median and range and qualitative variables as absolute and percentage frequency. Chi square test (or Fisher exact test when necessary) and Mann-Whitney non-parametric tests were used to compare categorical and quantitative un-paired data. Receiver operating characteristics (ROC) curves were constructed to assess the ability of IGF-I $\times$ ULN and Ki67 
to discriminate between responsive and not responsive patients, according to treatments. According to the primary objective, a prediction study was conducted, in order to identify the treatment outcome based on multiple variables/predictors. In fact, etiology studies uncover the casual effect on a specific risk factor on the outcome, adjusting for confounding factors that are selected on the basis of pre-existing knowledge of causal relations (18). Variables associated with treatment outcome at the univariate analysis were tested for possible influence on progression free survival using the KaplanMeier method and log-rank test. Risk factors found to have a $P<0.25$ at univariate analysis were included in the multivariate Cox regression survival analysis, as suggested by Hosmer and Lemeshow (19). Only observation with complete information was used for the analysis (complete case analysis). As prediction study, all variable were considered equal and were used collectively. None specific confounders were singled out, as the aim of a prediction study is not the identification of the effect single variable, but the prediction of the outcome though the combination of all predictors in the model (18). Results were expressed in terms of hazard ratio (HR) and 95\% CI. According to the prognostic nature of the study, sensitivity, specificity and area under the curve (AUC) were tested. The analyses were performed using SPSS software version 24.0 for Windows.

\section{Results}

A total of 74 patients entered the study. 48 patients were females (64.9\%). Median age at baseline was 38.5 years (IQR: 14, range: $18-74$ ). All patients had active acromegaly at baseline (median IGF-I $\times$ ULN: 3.3 IQR: 1.6; median GH: 27.5 IQR: $26.5 \mathrm{ng} / \mathrm{mL}$ ).

Before entering the study, all patients had undergone surgery, followed by medical therapy with first-generation SRLs. Dose of SRLs was up titrated as needed, including high dosage (Lanreotide ATG 120 mg every 3 weeks or Octreotide Lar 30 mg every 3 weeks) $(9,10,11,12,13,14$, $15,16,17,18,19,20)$. The baseline patients' features were similar among the two treatment groups, as summarized in Table 1.

Seven patients on treatment with Pegvisomant plus first-generation SRLs and five patients on treatment with Pasireotide LAR were reported in two previous studies of our research group $(14,21)$.

\section{Pegvisomant plus first-generation SRLs treatment group}

Forty-one patients were treated with Pegvisomant plus first-generation SRLs. At baseline, median IGF-I $\times$ ULN was 3.7 IQR: 1.4 and median GH was 18.5, IQR: $32 \mathrm{ng} / \mathrm{mL}$. At study entry, median dosage of Pegvisomant was $15 \mathrm{mg} /$ daily (minimum: $10 \mathrm{mg} /$ daily, maximum: $20 \mathrm{mg} /$ daily). The median duration of treatment was 54 months (range: 24-180 months). At the end of the study, biochemical control was reached in 35 patients $(85.4 \%)$. Tumor shrinkage did not occur in any case. Median Pegvisomant dose was $15 \mathrm{mg} /$ daily (minimum: $10 \mathrm{mg} /$ daily, maximum: $30 \mathrm{mg} /$ daily) in patients who reached acromegaly control and of $30 \mathrm{mg} /$ daily (minimum: $30 \mathrm{mg} /$ daily, maximum: $40 \mathrm{mg} /$ daily) in patients with persistently active disease.

Table 2 summarizes clinical and molecular features of patients treated with Pegvisomant, according to the treatment outcome. At the univariate analysis, notresponsive patients showed higher pre-treatment IGF-I level $(P=0.008)$ and higher value of Ki67 $(P=0.001)$. The area under the ROC curve developed for Ki67 and for IGF-I $\times$ ULN were respectively 0.697 and 0.855 . Optimal cut-off values were identified at Ki- $67>4 \%$ (specificity of $100 \%$ and a sensitivity of $50 \%$ ) and at IGF-I $>3.3 \times$ ULN (specificity of $78.8 \%$ and a sensitivity of $83.3 \%$ ). All six cases resistant to treatment with Pegvisomant had a Ki-67 $>4 \%$. The high grade of cavernous sinus invasion (Grade 4 of Knosp score) and the tumor extension to the third ventricle are associated with a poor response to Pegvisomant (respectively $P=0.01$ and $P=0.002$ ) (Table $3)$. These results were confirmed also by the Cox regression and survival analysis that showed a poor treatment response and a shorter progression free time in cases with tumor extension to the third ventricle $(P=0.004)$, with a Ki67 Li higher than $4 \%(P=0.004)$ and of the IGF-I value $\times$ ULN higher than $3.3(P=0.03)$ in predicting the response to Pegvisomant (Figs 1A and 2, Table 3). The survival analysis proved also a shorted progression free time in tumors with high grade of cavernous sinus invasion (grade 4 of Knosp score), as shown in Fig. 2.

\section{Pasireotide LAR treatment group}

Thirty-three patients were treated with Pasireotide LAR. At study entry, median IGF-I $\times$ ULN was 2.1 (IQR: 1.4 ) and median GH was 7 (IQR: $5.6 \mathrm{ng} / \mathrm{mL}$ ). 15 patients were treated with Pasireotide LAR $40 \mathrm{mg} / \mathrm{monthly}$ and the 
Table 1 Clinical aspects of study population. Univariate analysis. Data are presented as n (\%) or as median (IQR).

\begin{tabular}{l}
\hline \\
\hline Patients, $n$ \\
Gender \\
Females \\
Males \\
Age at acromegaly diagnosis \\
GH at acromegaly diagnosis ng/mL \\
IGF-I $\times$ ULN at acromegaly diagnosis \\
Tumor dimension \\
Macroadenoma \\
Tumor max diameter, mm \\
Invasion of cavernous sinus \\
Knosp score in cavernous sinus invasive \\
cases (49 pts) \\
Grade 3 \\
Grade 4 \\
Third ventricle extension \\
Post-surgical GH \\
Post-surgical IGF-I $\times$ ULN \\
Size of post-surgical residual, mm \\
Ki67 Li \% \\
Age at baseline \\
GH at baseline \\
IGF-I $\times$ ULN at baseline \\
Resistance to first-generation SRLs \\
Partial \\
Complete \\
Diabetes at baseline \\
Glycated hemoglobin* \\
Diabetes treatment \\
Hypocaloric diet \\
OHG drugs \\
Insulin \\
OHG drug plus insulin \\
\end{tabular}

\begin{tabular}{c} 
All patients \\
\hline 74 \\
$48(64.9 \%)$ \\
$26(35.1 \%)$ \\
$38.5(14)$ \\
$27.5(26.5)$ \\
$3.3(1.6)$ \\
$74(100 \%)$ \\
$37.5(17)$ \\
$49(66.2 \%)$
\end{tabular}

\begin{tabular}{c}
\hline Peg-V plus first-generation SRLs \\
\hline 41 \\
$28(68.3 \%)$ \\
$13(31.7 \%)$ \\
$37(27)$ \\
$18.5(32)$ \\
$3.7(1.4)$ \\
$41(100 \%)$ \\
$37(21)$ \\
$27(55.1 \%)$
\end{tabular}

\begin{tabular}{|c|c|}
\hline Pasi-LAR group & P-value \\
\hline 33 & $\begin{array}{r}N A \\
0.491\end{array}$ \\
\hline \multicolumn{2}{|l|}{$20(60.6 \%)$} \\
\hline $36(23.4)$ & 0.815 \\
\hline $20(30)$ & 0.749 \\
\hline 3.4 (1.6) & 0.189 \\
\hline $33(100 \%)$ & NA \\
\hline 37.5 (22.5) & 0.871 \\
\hline \multirow[t]{2}{*}{22 (44.9\%) } & 0.941 \\
\hline & 0.08 \\
\hline \multicolumn{2}{|l|}{$6(27.3 \%)$} \\
\hline \multicolumn{2}{|l|}{$16(72.7 \%)$} \\
\hline $2(40 \%)$ & 0.983 \\
\hline $11.8(19)$ & 0.340 \\
\hline $1.9(1.1)$ & 0.861 \\
\hline $8(7)$ & 0.478 \\
\hline $2(1)$ & 0.565 \\
\hline $43(20)$ & 0.955 \\
\hline $7.0(5.6)$ & 0.776 \\
\hline \multirow[t]{2}{*}{$2.1(1.4)$} & 0.355 \\
\hline & 0.678 \\
\hline \multicolumn{2}{|l|}{$13(31.7 \%)$} \\
\hline \multicolumn{2}{|l|}{$28(68.3 \%)$} \\
\hline $8(42.1 \%)$ & 0.184 \\
\hline \multirow[t]{2}{*}{$6.1(0.5)$} & 0.201 \\
\hline & 0.822 \\
\hline \multicolumn{2}{|l|}{2 (66.7\%) } \\
\hline \multicolumn{2}{|l|}{$5(62.5 \%)$} \\
\hline \multicolumn{2}{|l|}{$2(66.7 \%)$} \\
\hline $2(40 \%)$ & \\
\hline
\end{tabular}

*The value of glycated hemoglobin was referred to diabetic patients. OHG, oral hypoglycemic.

remaining 18 patients with $60 \mathrm{mg} /$ monthly. The median duration of treatment was of 37 months (range: 15-86 months). At the end of the study, 23 patients achieved biochemical control (69.7\%). Tumor shrinkage did not occur in any case. Of the 23 controlled patients at the end of the study, 10 patients were on treatment with Pasireotide LAR $40 \mathrm{mg} / \mathrm{monthly}$ and the remaining 13 patients with Pasireotide LAR $60 \mathrm{mg} / \mathrm{monthly.} \mathrm{Instead,}$ among the 10 patients with active acromegaly, all patients were on treatment with Pasireotide Lar $60 \mathrm{mg} / \mathrm{monthly.}$

Table 4 summarizes the clinical and molecular features of patients treated with Pasireotide LAR, according to the treatment outcome. At the univariate analysis, not responsive patients showed higher pre-treatment IGF-I level $(P=0.04)$, tumor extension to the third ventricle $(P=0.03)$, absent/low membranous expression of SST5 $(P=0.02)$ and carried more frequently the d3-isoform of the GHR $(P=0.003)$ (Table 5).
The area under the ROC curve developed for IGF-I $\times$ ULN was 0.691 . Optimal cut-off value was identified at IGF-I > $2.3 \times$ ULN (specificity: 70\%; sensitivity: 74.9\%).

These results were confirmed by the Cox regression and survival analysis that showed a poor response to Pasireotide LAR and a shorter progression free time in cases with tumor extension to the III ventricle, IGF-I value $\times$ ULN higher than 2.3 , with an absent/low SST5 membranous expression (Volante score $0-1$ ) and in patients carrying the d3-deleted GHR isoform, as shown in Figs 1B and 3, Table 5).

\section{Discussion}

Personalized therapy for aggressive acromegaly represents an open issue. Recently, molecular biomarkers of responsiveness to first-generation SRLs were validated 
Table 2 Clinical aspect of patients responsive and not-responsive to Pegvisomant plus first-generation SRLs. Data are presented as $n(\%)$ or as median (IQR). Quantitative variables were expressed as median and range and qualitative variables as absolute and percentage frequency.

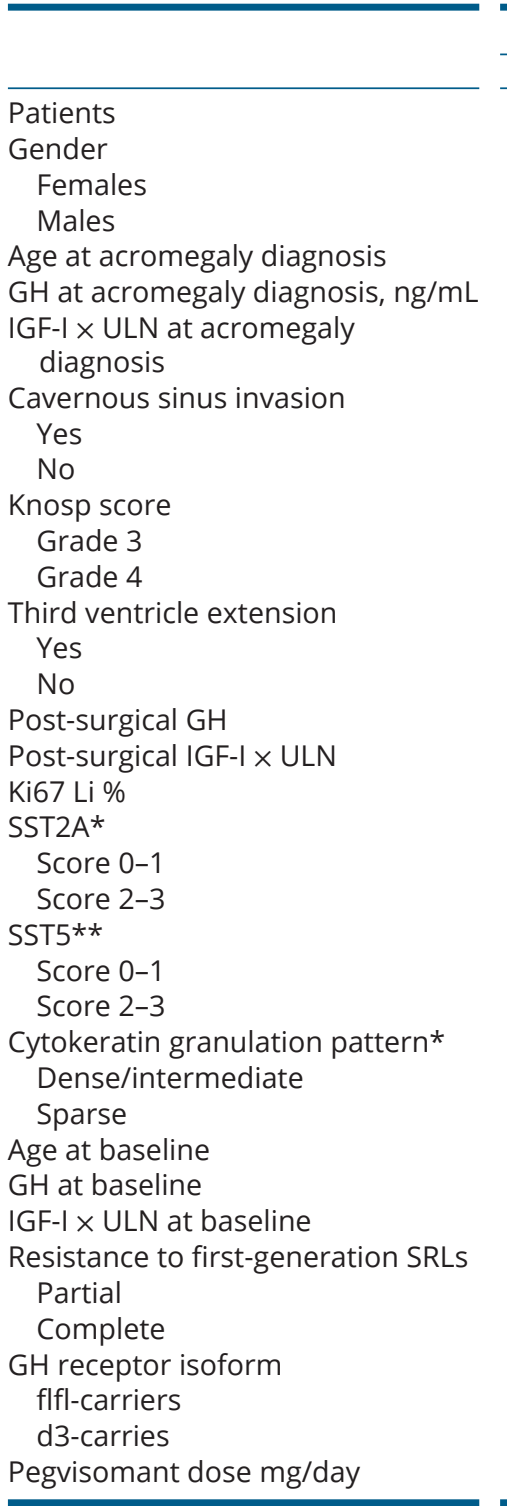

\begin{tabular}{|c|c|}
\hline \multicolumn{2}{|c|}{ Peg-V } \\
\hline Responsive & Not-responsive \\
\hline 35 (85.4\%) & $6(14.6 \%)$ \\
\hline 23 (65.7\%) & $5(83.3 \%)$ \\
\hline 12 (34.3\%) & $1(16.7 \%)$ \\
\hline $31(23.5)$ & $25(44)$ \\
\hline $17(29)$ & $15(40)$ \\
\hline $3.7(1.7)$ & $4.1(1.3)$ \\
\hline
\end{tabular}

\begin{tabular}{c}
$\begin{array}{c}\text { Sensitivity \% } \\
(95 \% \mathrm{Cl})\end{array}$ \\
\hline NA
\end{tabular}

$\begin{gathered}\text { Specificity \% } \\ (95 \% \mathrm{Cl})\end{gathered}$

$82.1(66.5-91.8)$

$7.7(2.1-21.5)$

NA

$22(62.9 \%)$

$5(83.3 \%)$

$13(37.1 \%)$

$1(16.7 \%)$

$15(68.2 \%)$

$7(31.8 \%)$

$0(0 \%)$

$5(100 \%)$

$0(0 \%)$

$35(100 \%)$

$3(50 \%)$

$3(50 \%)$

$7.1(12)$

$1.4(0.6)$

$2(1.5)$

$24(8)$

$2.0(3)$

$3.5(5)$

$5(27.8 \%)$

$13(72.2 \%)$

$2(75 \%)$

$1(25 \%)$

$5(33.3 \%) \quad 1(25 \%)$

$10(66.7 \%)$

$6(33.3 \%)$

$12(66.7 \%)$

$41(19)$

5 (14)

$1.9(1.4)$

$13(37.1 \%)$

$22(62.9 \%)$

$19(76 \%)$

$6(24 \%)$

20 (10)
$2(75 \%)$

0

$3(100 \%)$

42 (44)

$10(9)$

$3.5(1.1)$

0

$6(100 \%)$

$2(33.3 \%)$

$4(66.7 \%)$

30 (10)
NA

NA

NA

81.5 (65.7-91.3)

$100(84.5-100)$

$6(0-10.7)$

NA

NA

NA

71.4 (47.7-87.8)

83.3 (57.7-95.6)

$16.7(4.4-42.3)$

NA

$100(81.5-100)$

$20(6.8-43.6)$

NA

NA

NA

NA

$100(89.3-100)$

NA

NA

$0.5(0.1-0.8)$

$0.7(0.3-1)$

$0.7(0.5-0.9)$

$7.1(2-20.8)$

NA

NA

$7.9(2.2-21.7)$

NA

90.5 (73.3-97.5)

40 (23.5-58.9)

NA

NA

NA

$0.9(0.8-0.9)$

*Data available for 21 cases; **Data available for 18 cases.

AUC, area under the curve.

in a retrospective study, confirming the prognostic role of Ki-67, SST2 and E-cadherin (22). In this study, we investigated the molecular and clinical biomarkers able to predict the responsiveness to second line therapies, in acromegaly patients resistant to first-generation SRLs.

The treatment with Pegvisomant in association with first-generation SRLs may allow the biochemical control of the disease in $50-100 \%$ of treated patients $(21,23$, $24,25,26,27,28)$. The efficacy of Pasireotide LAR was reported between $37 \%$ and $55 \%$ of acromegaly patients, mostly resistant to first-generation SRLs $(14,29,30,31$, 32, 33).

Based on the high efficacy of Pegvisomant, many studies investigated the factors associated to the dose required to normalize IGF-I, rather than factors potentially associated to biochemical control of acromegaly. In a recent review, Bernabeu et al. showed that higher dose of Pegvisomant are required in females, in patients with higher body weight and in patients with more aggressive acromegaly $(34,35)$. 
Table 3 Factors associated to responsiveness to Pegvisomant. Univariate and multivariate model.

\begin{tabular}{|c|c|}
\hline & $P$-value \\
\hline Gender & 0.368 \\
\hline Age at acromegaly diagnosis & 0.493 \\
\hline $\mathrm{GH}$ at acromegaly diagnosis ng/mL & 0.364 \\
\hline IGF-I $\times$ ULN at acromegaly diagnosis & 0.341 \\
\hline Cavernous sinus invasion & 0.317 \\
\hline Grade 4 of Knosp score & 0.01 \\
\hline Third ventricle extension & 0.002 \\
\hline Post-surgical GH & 0.327 \\
\hline Post-surgical IGF-I × ULN & 0.217 \\
\hline $\mathrm{Ki} 67 \mathrm{Li}>4 \%$ & $<0.001$ \\
\hline SST2A & 0.247 \\
\hline SST5 & 0.73 \\
\hline Cytokeratin pattern & 0.342 \\
\hline Age at baseline & 0.854 \\
\hline $\mathrm{GH}$ at baseline & 0.555 \\
\hline IGF-I >3.3 × ULN at baseline & 0.01 \\
\hline Resistance to first-generation SRLS & 0.08 \\
\hline GH receptor isoform & 0.07 \\
\hline Pegvisomant dose median mg/day (IQR) & 0.008 \\
\hline
\end{tabular}

In this study, we found that patients who did not reach the biochemical control of acromegaly with Pegvisomant plus first-generation SRLs had aggressive disease in terms of hormonal secretion, extension and proliferation of the pituitary tumor. In fact, according to our data, an IGF-I value higher than $3.3 \times$ ULN can predict the poor outcome

\begin{tabular}{|c|c|c|}
\hline \multicolumn{2}{|c|}{ Peg-V } & \multirow[b]{2}{*}{$\mathrm{HR}(95 \% \mathrm{Cl})$} \\
\hline $\mathrm{HR}(95 \% \mathrm{Cl})$ & Adj $P$-value & \\
\hline $0.38(0.04-3.7)$ & NA & NA \\
\hline NA & NA & NA \\
\hline NA & NA & NA \\
\hline NA & NA & NA \\
\hline $2.9(0.3-28.1)$ & NA & NA \\
\hline $1.7(1.1-2.7)$ & 0.09 & NA \\
\hline $2(1.9-4.4)$ & 0.004 & $1.6(1.2-4.6)$ \\
\hline NA & NA & NA \\
\hline NA & NA & NA \\
\hline $2(1.1-5.3)$ & 0.004 & $3.9(1.4-4)$ \\
\hline $0.19(0.01-2.6)$ & NA & NA \\
\hline $1(0.07-13.8)$ & NA & NA \\
\hline $1.25(0.9-1.6)$ & NA & NA \\
\hline NA & NA & NA \\
\hline NA & NA & NA \\
\hline $9.5(1.3-70.9)$ & 0.03 & $1.3(1.1-6)$ \\
\hline $1.3(1.1-1.5)$ & NA & NA \\
\hline $6.3(0.9-43.6)$ & NA & NA \\
\hline NA & NA & NA \\
\hline
\end{tabular}

to Pegvisomant. To our knowledge, our study investigated for the first time the role of Ki67 in predicting Pegvisomant responsiveness. A Ki67 proliferative index higher than $4 \%$ seem able to discriminate cases responsive and nonresponsive, with high specificity and sensitivity. It is well known that the Ki67 is a marker of proliferation of the

Adj. HR (95\%CI); p-value

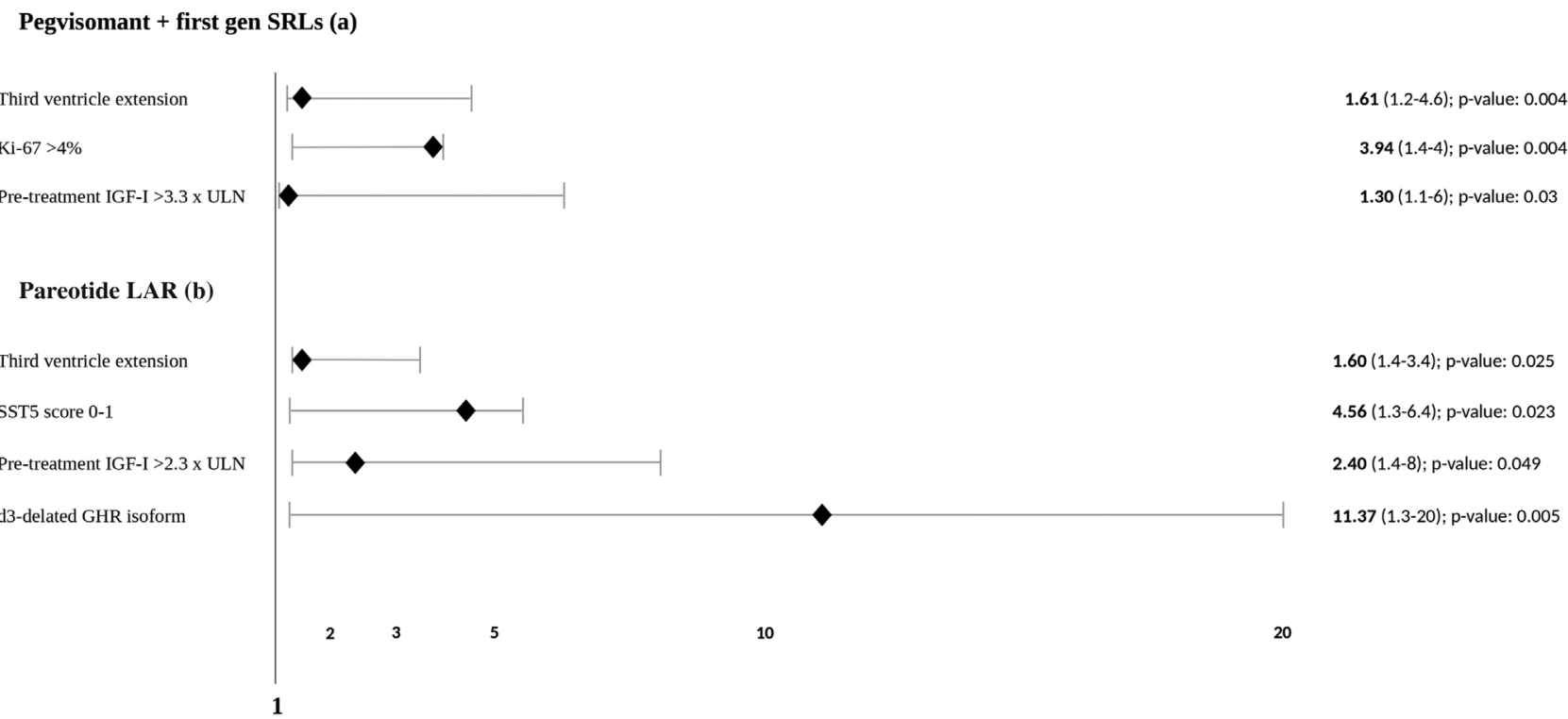

\section{Figure 1}

Clinical and molecular biomarkers of response to Pegvisomant plus first-generation SRLs and Pasireotide LAR. Forrest plot represents the hazard ratio of factors associated with the responsiveness to treatment with Pegvisomant plus first-generation SRLs (A) and with Pasireotide LAR (B). The statistical analysis was conducted with a Cox logistic regression. 


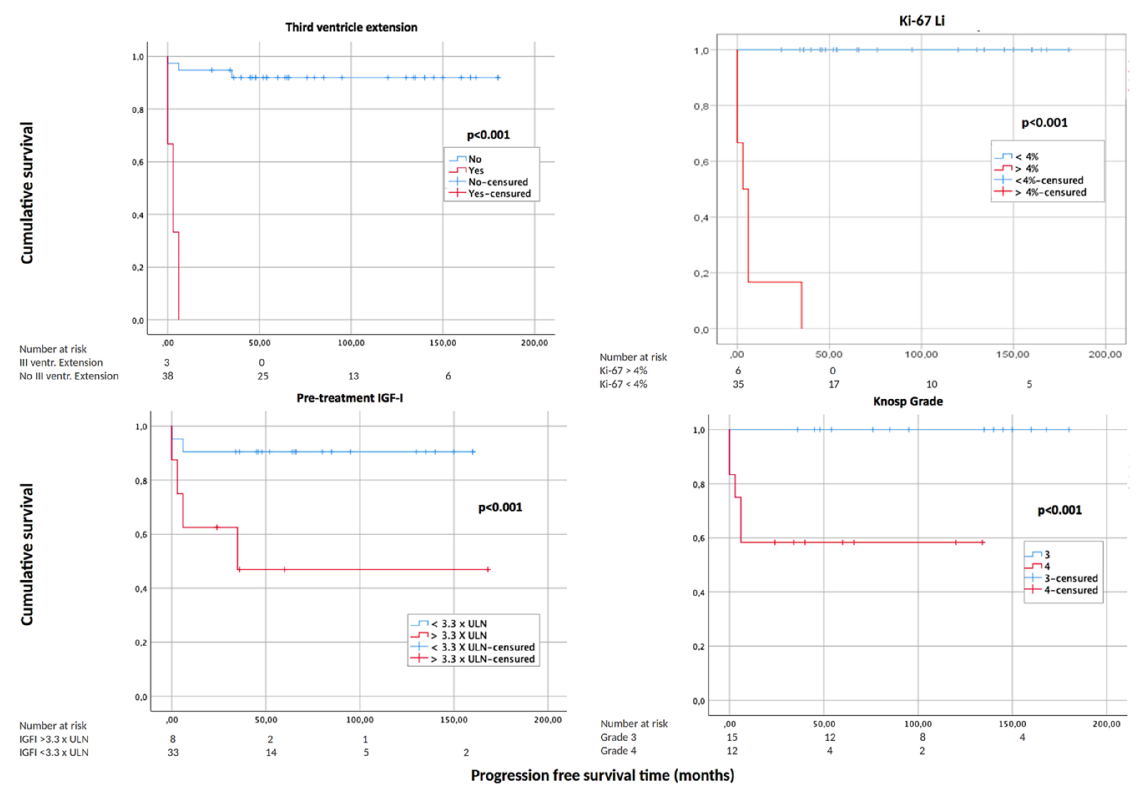

\section{Figure 2}

Survival analysis. Progression free disease time in patients on treatment with

Pegvisomant plus first-generation SRLS. tumor cells, as it is expressed during the interphase of the cell cycle. Higher Ki67 Li was reported in patients resistant to first-generation SRLs as compared to responsive ones $(35,36)$ and to those cured only through surgery (35). In addition, the pituitary tumors extended to the third ventricle and totally encased the intra-cavernous carotid artery (Grade 4 according to Knosp classification) in all patients not-responsive to Pegvisomant.

Limited and controversial data are available on predictors of response to Pasireotide LAR, in particular on the role of SST5. In this series, we found that all patients who achieved acromegaly control carried a membranous expression of the SST5 in tumoral tissue (score 2 and 3 of Volante classification) (15), confirming in this larger cohort the results of our previous study (14). In our previous study, in fact, we found that the immunohistochemistry detection of the SST5 was a predictor of the outcome to Pasireotide LAR treatment, in patients resistant to first-generation SRLs and that the high SST5 expression was associated with a greater reduction of IGF-I during Pasireotide LAR treatment (14). Similar results were demonstrated by an 'in-vitro' study showing that the lower SST2 and SST2/SST5 ratio at mRNA levels are associated to a better response to Pasireotide (37). Recently, Muhammad et al. (38) suggested instead that Pasireotide LAR responsiveness is driven by SST2 rather than by SST5. Data from these clinical studies are difficult to compare due to the heterogeneous study population, in particular in terms of selection of patients responsive or resistant to first-generation SRLs. In addition, the variable reports on SST5 may be explained by the variability of techniques in performing immunohistochemistry, by the heterogeneity in tumor SST expression in different studies and by the pre-surgical medical treatment with SRLs (39, $40,41)$. In this study and in our clinical practice, we adopt the Volante et al score system (15) for investigating the expression of the SST receptors. Although Volante et al. score considers both membrane and non-membrane, cytoplasm and even nuclear staining, this scoring system accurately reflects the true receptor cell distribution and internalization. All these features are observed in neuroendocrine neoplasm cells and clearly seen with the 'more modern UMB antibodies'. However, the Volante score strongly emphasizes the membrane staining vs the other types of staining-features that all accurately mirror cell differentiation.

Interestingly, in this study we found that four of the six patients resistant to Pasireotide LAR showed a high expression of SST5. Although these patients did not achieve acromegaly control, they experienced a higher reduction in IGF-I levels, as compared to cases with low/ absent SST5 expression (IGF-I reduction, respectively, of -1.3 vs 0.05$)$. In addition, none of the patients with absent/low expression of SST5 responded to Pasireotide LAR, consistent with our previous study (14). Similarly to those observed in the group of patients treated with Pegvisomant, the tumor extension to the third ventricle was associated to the absence of response to Pasireotide LAR, as well as the IGF-I values (tested before starting Pasireotide LAR) higher than $2.3 \times$ ULN.

Here, we have investigated for the first time the role of the GHR isoform in predicting the outcome of 
Table 4 Clinical aspect of patients responsive and not-responsive to Pasireotide LAR. Data are presented as $n$ (\%) or as median (IQR).

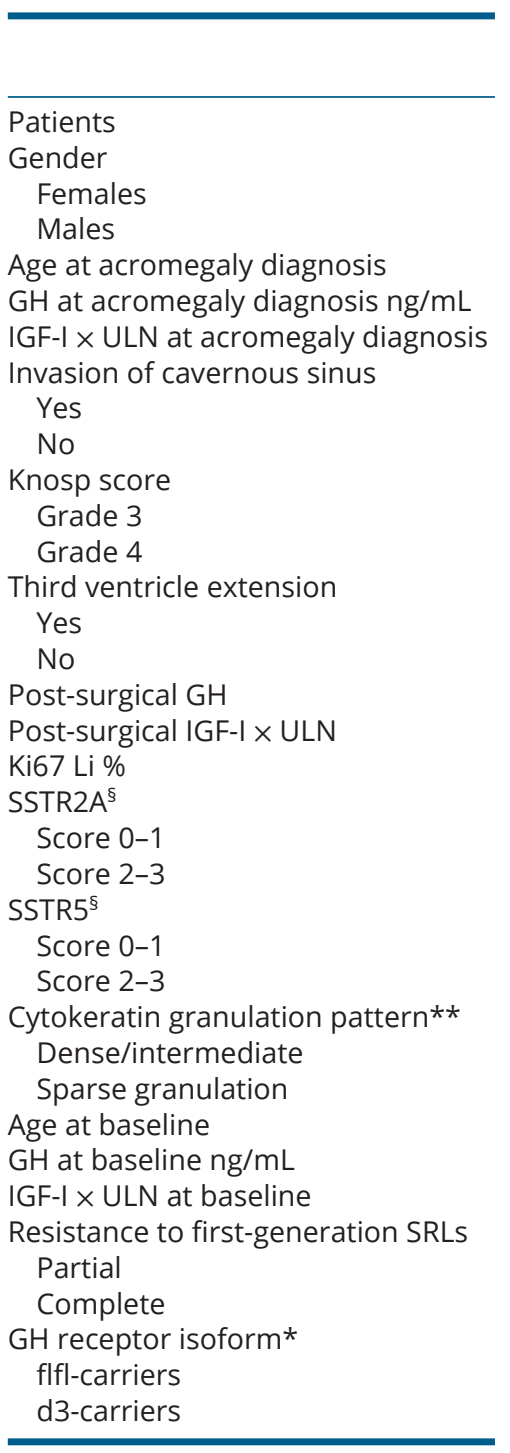

\begin{tabular}{|c|c|}
\hline \multicolumn{2}{|c|}{ Pasi-Lar } \\
\hline Responsive & Not-responsive \\
\hline $23(69.7 \%)$ & $10(30.3 \%)$ \\
\hline 13 (56.5\%) & $7(70 \%)$ \\
\hline $10(43.5 \%)$ & $3(30 \%)$ \\
\hline $43(19)$ & $40.5(32)$ \\
\hline $18(27.5)$ & $25(25)$ \\
\hline $3(1.5)$ & 4 (1.9) \\
\hline $16(69.6 \%)$ & $6(60 \%)$ \\
\hline $7(30.4 \%)$ & $4(40 \%)$ \\
\hline $6(37.5 \%)$ & 0 \\
\hline $10(62.5 \%)$ & $6(100 \%)$ \\
\hline $0(0 \%)$ & $2(20 \%)$ \\
\hline $23(100 \%)$ & $8(80 \%)$ \\
\hline 15 (13) & $8.5(18)$ \\
\hline $1.3(1.4)$ & $2(1.8)$ \\
\hline $2.2(1.1)$ & $3(3.5)$ \\
\hline $7(53.8 \%)$ & 2 (33.3\%) \\
\hline $6(46.2 \%)$ & $4(66.7 \%)$ \\
\hline 0 & $2(33.3 \%)$ \\
\hline $13(100 \%)$ & $4(66.7 \%)$ \\
\hline $4(36.4 \%)$ & $1(16.7 \%)$ \\
\hline $7(63.6 \%)$ & $5(83.3 \%)$ \\
\hline $43(19)$ & $41(32)$ \\
\hline $5(8)$ & $8(3)$ \\
\hline $1.9(1.2)$ & $2.6(1.7)$ \\
\hline $5(21.7 \%)$ & $4(40 \%)$ \\
\hline $18(78.3 \%)$ & $6(60 \%)$ \\
\hline $14(77.8 \%)$ & $1(12.5 \%)$ \\
\hline $4(22.2 \%)$ & 7 (87.5\%) \\
\hline
\end{tabular}
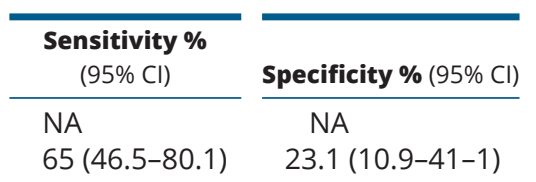

\begin{tabular}{l} 
AUC $(95 \% \mathrm{Cl})$ \\
\hline NA \\
NA
\end{tabular}

NA
NA
NA

NA

NA

NA

$72.7(54.2-86.1)$

$36.4(21-54.9)$

$100(81.5-100)$

$37.5(18.9-60.2)$

NA

$80(65.4-91.2)$

$25(12.9-44.3)$

NA

NA
NA
NA

NA

NA

$77.6(52.7-92.4)$

NA

$40(19.5-64.2)$

$0.4(0.2-0.7)$

$0.6(0.4-0.8)$

0.7 (0.5-0.9)

NA

$$
12(0-20.9) \quad 23.5(8.4-48.6) \quad \text { NA }
$$

$80(53.3-94.1)$

$41.7(19.8-67)$

NA

$\begin{array}{lll}\text { NA } & \text { NA } & 0.2(0-0.5) \\ \text { NA } & \text { NA } & 0.8(0.6-1) \\ \text { NA } & \text { NA } & 0.9(0.7-1) \\ 55.6(37.5-72.3) & 25(12.3-43.4) & \text { NA }\end{array}$

93.9 (74.7-99)

$63.6(42.7-80.7)$

NA

*Data available for 26 cases; $^{\S}$ Data available for 19 patients; **Data available for 17 patients.

AUC, area under the curve.

medical treatment with Pasireotide LAR. The GHR is a transmembrane protein and is constituted by two dimers. The GHR gene consists of nine exons encoding the receptor protein and several introns (42). The full-length or the d3-deleted dimers differ respectively for the retention or exclusion of the exon 3 (42). As for consequence the GHR can be constituted by homo- or hetero-dimers for full-length or d3-deleted isoform (43). The d3-deleted isoform is dominant over the full-length one (42) and enhances GHR signal transduction by altering the structure of the extracellular domain of the receptor. The increased intracellular signal transduction may increase the sensitivity to GH: GH-deficient children carried the d3-GHR have an increased growth velocity and a higher final height (44) when treated with recombinant human $\mathrm{GH}$ (rhGH). In adult GHD patients, d3-GHR influences positively also metabolic and bone effects of rhGH (17, 45). On the other hand, d3-GHR polymorphism confers higher susceptibility to $\mathrm{GH}$ stimulation in patients with acromegaly and may negatively affect phenotypic presentation and long-term complications of the disease, as showed in bone metabolism (46). The prognostic role of GHR isoform in acromegaly patients according to the different medical therapies remain still controversial, in particular in those on treatment with Pegvisomant.

In a recent meta-analysis, Frank et al. reviewed previous clinical studies that investigated the possible effect of the GHR polymorphism and ruled out a possible biochemical 
Table 5 Factors associated to responsiveness to Pasireotide LAR. Univariate and multivariate model.

\begin{tabular}{|c|c|c|c|c|}
\hline & \multicolumn{4}{|c|}{ Pasireotide LAR } \\
\hline & $P$-value & $\mathrm{HR}(95 \% \mathrm{Cl})$ & Adj. $P$-value & $\mathrm{HR}(95 \% \mathrm{Cl})$ \\
\hline Gender & 0.371 & $0.56(0.1-2.7)$ & NA & NA \\
\hline Age at acromegaly diagnosis & 0.564 & NA & NA & NA \\
\hline $\mathrm{GH}$ at acromegaly diagnosis, ng/mL & 0.288 & NA & NA & NA \\
\hline IGF-I $\times$ ULN at acromegaly diagnosis & 0.069 & NA & NA & NA \\
\hline Invasion of cavernous sinus & 0.440 & $0.6(0.1-3)$ & NA & NA \\
\hline Knosp score & 0.107 & $0.6(0.4-0.9)$ & NA & NA \\
\hline Third ventricle extension & 0.03 & $1.2(1.1-1.7)$ & 0.025 & $1.6(1.4-3.4)$ \\
\hline Post-surgical GH & 0.99 & $\mathrm{NA}$ & NA & NA \\
\hline Post-surgical IGF-I × ULN & 0.247 & NA & NA & NA \\
\hline Ki67 Li \% & 0.424 & NA & NA & NA \\
\hline SSTR2A score & 0.37 & $1.8(0.3-11.1)$ & NA & NA \\
\hline SSTR5 score & 0.02 & $4.2(1.8-10)$ & 0.023 & $4.56(1.3-6.4)$ \\
\hline Cytokeratin pattern & 0.395 & $2.8(0.3-33.9)$ & NA & NA \\
\hline Age at baseline & 0.603 & NA & NA & NA \\
\hline $\mathrm{GH}$ at baseline & 0.288 & NA & NA & NA \\
\hline IGF-I > 2.3 × ULN at baseline & 0.04 & $1.2(1.1-1.7)$ & 0.049 & $2.4(1.4-8)$ \\
\hline Resistance to first-generation SRLs & 0.252 & $1.8(0.6-4.8)$ & NA & NA \\
\hline GH receptor isoform & 0.003 & $2.5(1.1-5.7)$ & 0.005 & $11.37(1.3-20)$ \\
\hline
\end{tabular}

Adj., adjusted; HR, hazard ratio.

effect on long term treatment with Pegvisomant (45). However, in our previous experience (47), we found that d3-GHR carriers need lower Pegvisomant dose and a shorter treatment duration to normalize IGF-I levels, at least during the first year of treatment. We hypothesize that this phenomenon may be due to a progressive receptor saturation mechanism due to the increase of the circulating $\mathrm{GH}$ (47). A potential positive effect of the d3-GHR may be considered during the first year of therapy. Our results here confirmed the absence of an effect of GHR polymorphism in long term treatment with Pegvisomant (median follow-up 54 months), but it doesn't exclude a short term effect.

In this study, we also demonstrated that d3-GHR carriers more frequently did not reach biochemical control during Pasireotide LAR treatment (Fig. 4A and Table 4). Instead, flfl-GHR carriers have been good Pasireotide LAR responders, despite similar values of GH and IGF-I before starting Pasireotide LAR treatment (Fig. 4B and C). This effect may be due to the previous described high sensitivity of the d3-deleted GHR isoform to GH stimulation, as shown also during treatment with firstgeneration SRLs.

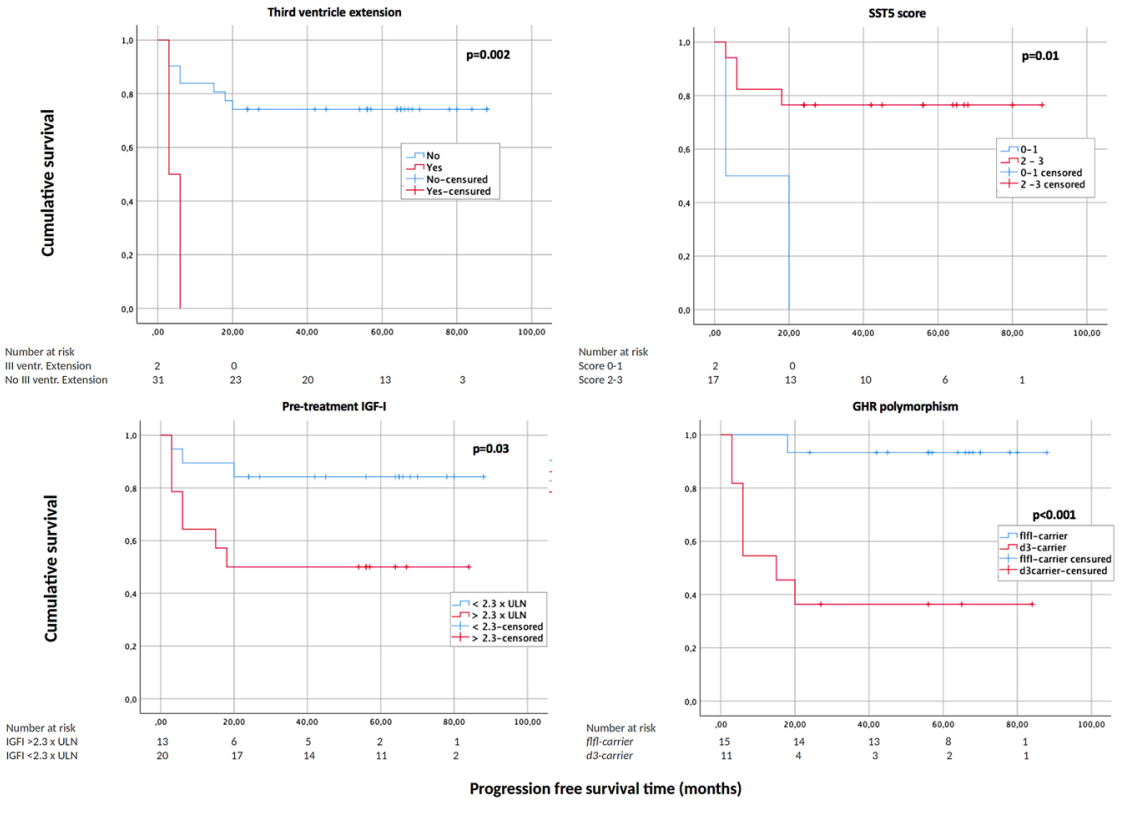

Figure 3

Survival analysis. Progression-free disease time in patients on treatment with Pasireotide LAR. 
In addition, in this study, we investigated a possible correlation between the GHR isoform and the tumor aip expression, without finding significantly difference (aip expression in d3-delated GHR isoform: 140; IQR: 198; aip expression in flfl- GHR isoform: 180; IQR: $195, P=0.844$ ). In this cohort, three patients were affected by genetic acromegaly: two patients carried mutation of GNAS gene (R201C and Q227L) and one carried mutation of the aip gene (c.760T>C). D3-delated GHR isoform was detected in the patients with GNAS R201C mutation. Instead the other two cases carried the flfl-GHR. All patients were responsive to combination treatment with Pegvisomant and first-generation SRLs.

The main limitation of our research is the small sample size, in particular when the study population is stratified for treatment and outcome. This limitation may reduce the power of same tests, with the risk of false positive results. In addition, this study has a retrospective design and lacks of a randomization for the treatment groups. However, this study did not aim to compare the two-treatment cohorts per se, but it describes a real-life experience on a homogenous cohort of patients affected by difficult to control acromegaly resistant to first-generation SRLs, with a long-term follow-up. At least, this study did not allow us to provide data on the prognostic role of the cytokeratin pattern in responsiveness to Pasireotide LAR and Pegvisomant, similarly to other reports (48). This may be due to the nature of this cohort that represents a homogenous group of aggressive acromegaly disease, with a high prevalence of sparsely granulated GH secreting pituitary tumors.

To our knowledge, this study for the first time described biomarkers able to predict the responsiveness

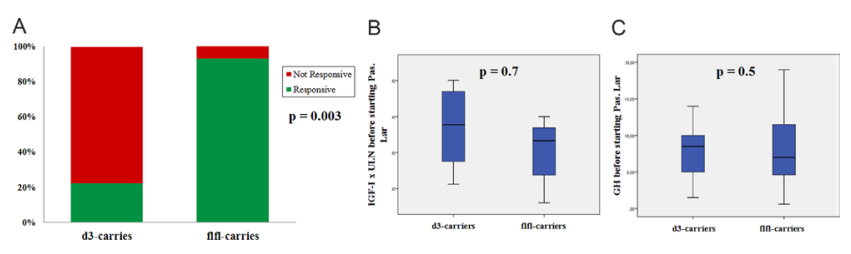

\section{Figure 4}

Response to Pegvisomant plus first-generation SRLs and Pasireotide LAR according to GHR polymorphism. (A) Histogram represents the outcome of treatment with Pasireotide LAR, according to GHR isoforms. The statistical analysis was conducted with Chi-square test. Box plots represent the median (IQR) of GH (B) and IGF-I $\times$ ULN (C) before starting treatment with Pasireotide LAR, according to GHR isoforms. The statistical analysis was with Mann-Whitney test. to Pasireotide LAR and Pegvisomant plus first-generation SRLs. Our data are in line with new WHO guidelines (6), where tumor proliferative potential measurements, and other clinical parameters such as tumor invasion, are strongly recommended to evaluate a potentially clinically aggressive adenoma.

In conclusion, our data suggest that available guidelines can be improved by including a combination of specific biochemical cut-offs and a panel of molecular tumor and peripheral markers including Ki67, somatostatin receptors and GHR polymorphism. Individualized treatment of patients with resistant acromegaly to first-generation SRLs may avoid further delays in controlling the disease and unnecessary side effects which may heavily impact on quality of life and patients' survival.

\section{Declaration of interest}

$\mathrm{M} F$ reports serving as an investigator with research grants to OHSU for Crinetics, Chiasma, Ionis, Novartis and serving as an occasional consultant to Crinetics, Chiasma, Ipsen, Novartis, Pfizer; A G: Research Grants from Ipsen, Novartis and Pfizer Consultant for Genevant and Ipsen.

\section{Funding}

This research did not receive any specific grant from any funding agency in the public, commercial or not-for-profit sector.

\section{Ethical approval}

All procedures performed in studies involving human participants were in accordance with the ethical standards of the institutional review board and with the 1964 Helsinki declaration and its later amendments or comparable ethical standards. The study was approved by Institutional Review Board of the Gemelli Hospital, Catholic University of the Sacred Heart, Rome. All patients entered the study signed an informed consensus.

\section{References}

1 Melmed S, Bronstein MD, Chanson P, Klibanski A, Casanuela FF, Wass JAH, Strasburger CJ, Luger A, Clemmons DR \& Giustina A. A consensus statement on acromegaly therapeutic outcomes. Nature Reviews: Endocrinology 201814 552-561. (https://doi.org/10.1038/ s41574-018-0058-5)

2 Holdaway IM, Bollard MJ \& Gamble GD. A meta-analysis of the effect of lowering serum levels of GH and IGF-I on mortality in acromegaly. European Journal of Endocrinology 2008159 89-95. (https://doi. org/10.1530/EJE-08-0267)

3 Molitch ME. Diagnosis and treatment of pituitary adenomas: a review. JAMA 2017317 516-524. (https://doi.org/10.1001/ jama.2016.19699)

4 Gadelha MR, Wildemberg LE, Bronstein MD, Gatto F \& Ferone D. Somatostatin receptor ligands in the treatment of acromegaly. Pituitary 201720 100-108. (https://doi.org/10.1007/s11102-0170791-0)

5 Gadelha MR, Kasuki L \& Korbonits M. Novel pathway for somatostatin analogs in patients with acromegaly. Trends in 
Endocrinology and Metabolism 201324 238-246. (https://doi. org/10.1016/j.tem.2012.11.007)

6 Lopes MBS. The 2017 World Health Organization classification of tumors of the pituitary gland: a summary. Acta Neuropathologica 2017 134 521-535. (https://doi.org/10.1007/s00401-017-1769-8)

7 Raverot G, Burman P, McCormack A, Heaney A, Petersenn S, popovic V, Trouillas J, Dekkers OM \& European Society of Endocrinology. European Society of Endocrinology Clinical Practice Guidelines for the management of aggressive pituitary tumours and carcinomas. European Journal of Endocrinology 2018178 G1-G24. (https://doi.org/10.1530/EJE-17-0796)

8 Dekkers OM \& Groenwold RHH. Study design: what's in a name? European Journal of Endocrinology 2020183 E11-E13. (https://doi. org/10.1530/EJE-20-0873)

9 Giustina A, Mazziotti G, Cannavò S, Castello R, Arnaldi G, Bugari G, Cozzi R, Ferone D, Formenti AM, Gatti E et al. High-dose and high-frequency lanreotide autogel in acromegaly: a randomized, multicenter study. Journal of Clinical Endocrinology and Metabolism 2017102 2454-2464. (https://doi.org/10.1210/jc.2017-00142)

10 Colao A, Auriemma RS, Lombardi G \& Pivonello R. Resistance to somatostatin analogs in acromegaly. Endocrine Reviews 201132 247-271. (https://doi.org/10.1210/er.2010-0002)

11 Knosp E, Steiner E, Kitz K \& Matula C. Pituitary adenomas with invasion of the cavernous sinus space: a magnetic resonance imaging classification compared with surgical findings. Neurosurgery 1993 33 610-617; discussion 617. (https://doi.org/10.1227/00006123199310000-00008)

12 Chiloiro S, Bianchi A, Doglietto F, de Waure C, Giampietro A, Fusco A, Iacovazzo D, Tartaglione L, Di Nardo F, Signorelli F et al. Radically resected pituitary adenomas: prognostic role of Ki 67 labeling index in a monocentric retrospective series and literature review. Pituitary 201417 267-276. (https://doi.org/10.1007/s11102013-0500-6)

13 Coli A, Asa SL, Fadda G, Scannone D, Chiloiro S, De Marinis L, Lauretti L, Ranelletti FO \& Lauriola L. Minichromosome maintenance protein 7 as prognostic marker of tumor aggressiveness in pituitary adenoma patients. European Journal of Endocrinology 2016 174 307-314. (https://doi.org/10.1530/EJE-15-0586)

14 Iacovazzo D, Carlsen E, Lugli F, Chiloiro S, Piacentini S, Bianchi A, Giampietro A, Mormando M, Clear AJ, Doglietto F et al. Factors predicting pasireotide responsiveness in somatotroph pituitary adenomas resistant to first-generation somatostatin analogues: an immunohistochemical study. European Journal of Endocrinology 2016 174 241-250. (https://doi.org/10.1530/EJE-15-0832)

15 Volante M, Brizzi MP, Faggiano A, La Rosa SL, Rapa I, Ferrero A, Mansueto G, Righi L, Garancini S, Capella C et al. Somatostatin receptor type $2 \mathrm{~A}$ immunohistochemistry in neuroendocrine tumors: a proposal of scoring system correlated with somatostatin receptor scintigraphy. Modern Pathology 200720 1172-1182. (https://doi. org/10.1038/modpathol.3800954)

16 Ali HR, Irwin M, Morris L, Dawson SJ, Blows FM, Provenzano E, Mahler-Araujo B, Pharoah PD, Walton NA, Brenton JD et al. Astronomical algorithms for automated analysis of tissue protein expression in breast cancer. British Journal of Cancer 2013108 602-612. (https://doi.org/10.1038/bjc.2012.558)

17 Mormando M, Chiloiro S, Bianchi A, Giampietro A, Angelini F, Tartaglione L, Nasto L, Milardi D, Formenti AM, Giustina A et al. Growth hormone receptor isoforms and fracture risk in adult-onset growth hormone-deficient patients. Clinical Endocrinology 201685 717-724. (https://doi.org/10.1111/cen.13161)

18 van Diepen M, Ramspek CL, Jager KJ, Zoccali C \& Dekker FW. Prediction versus aetiology: common pitfalls and how to avoid them. Nephrology, Dialysis, Transplantation 201732 ii1-ii5. (https://doi. org/10.1093/ndt/gfw459)

19 Hosmer DW \& Lemeshow S. Applied Logistic regression. New York: Wiley, 1989. (https://doi.org/10.1002/0471722146)
20 Mazziotti G, Porcelli T, Bogazzi F, Bugari G, Cannavò S, Colao A, Cozzi R, De Marinis L, degli Uberti E, Grottoli S et al. Effects of high-dose octreotide LAR on glucose metabolism in patients with acromegaly inadequately controlled by conventional somatostatin analog therapy. European Journal of Endocrinology 2011164 341-347. (https://doi.org/10.1530/EJE-10-0811)

21 Bianchi A, Valentini F, Iuorio R, Poggi M, Baldelli R, Passeri M, Giampietro A, Tartaglione L, Chiloiro S, Appetecchia M et al. Longterm treatment of somatostatin analog-refractory growth hormonesecreting pituitary tumors with pegvisomant alone or combined with long-acting somatostatin analogs: a retrospective analysis of clinical practice and outcomes. Journal of Experimental and Clinical Cancer Research 201332 40. (https://doi.org/10.1186/1756-9966-32-40)

22 Puig-Domingo M, Gil J, Sampedro Nuñez M, Jorda M, Webb SM, Serra G, Pons L, Salinas I, Blanco A, Marques-Pamies M et al. Molecular profiling for acromegaly treatment: a validation study. Endocrine-Related Cancer 202027 375-389. (https://doi.org/10.1530/ ERC-18-0565)

23 Neggers SJCMM, van Aken MO, Janssen JAMJL, Feelders RA, de Herder WW \& van der Lely AJ. Long-term efficacy and safety of combined treatment of somatostatin analogs and pegvisomant in acromegaly. Journal of Clinical Endocrinology and Metabolism 200792 4598-4601. (https://doi.org/10.1210/jc.2007-1234)

24 Trainer PJ, Ezzat S, D'Souza GA, Layton G \& Strasburger CJ. A randomized, controlled, multicentre trial comparing pegvisomant alone with combination therapy of pegvisomant and long-acting octreotide in patients with acromegaly. Clinical Endocrinology 2009 71 549-557. (https://doi.org/10.1111/j.1365-2265.2009.03620.x)

25 Jørgensen JOL, Feldt-Rasmussen U, Frystyk J, Chen JW, Kristensen LØ, Hagen C \& Ørskov H. Cotreatment of acromegaly with a somatostatin analog and a growth hormone receptor antagonist. Journal of Clinical Endocrinology and Metabolism 200590 5627-5631. (https://doi.org/10.1210/jc.2005-0531)

26 Ezzat S, Gaspo R, Serri O, Ur E \& Chik CL. A Canadian multi-centre, open-label long-term study of pegvisomant treatment in refractory acromegaly. Clinical and Investigative Medicine 200932 E265. (https:// doi.org/10.25011/cim.v32i6.10662)

27 Buchfelder M, van der Lely AJ, Biller BMK, Webb SM, Brue T, Strasburger CJ, Ghigo E, Camacho-Hubner C, Pan K, Lavenberg J et al. Long-term treatment with pegvisomant: observations from 2090 acromegaly patients in ACROSTUDY. European Journal of Endocrinology 2018179 419-427. (https://doi.org/10.1530/EJE-180616)

28 Leonart LP, Tonin FS, Ferreira VL, Fernandez-Llimos F \& Pontarolo R. Effectiveness and safety of pegvisomant: a systematic review and meta-analysis of observational longitudinal studies. Endocrine 2019 63 18-26. (https://doi.org/10.1007/s12020-018-1729-7)

29 Colao AAL, Bronstein MD, Brue T, De Marinis L, Fleseriu M, Guitelma M, Raverot G, Shimon I, Fleck J, Gupta P et al. Pasireotide for acromegaly: long-term outcomes from an extension to the Phase III (PAOLA) study. European Journal of Endocrinology 2020182583. (https://doi.org/10.1530/EJE-19-0762)

30 Gadelha M, Bex M, Colao A, Garcia EMPG, Poiana C, JimenezSanchez M, Yener S, Mukherjee R, Bartalotta A, Maamari R et al. Evaluation of the efficacy and safety of switching to pasireotide in patients with acromegaly inadequately controlled with firstgeneration somatostatin analogs. Frontiers in Endocrinology 202010 931. (https://doi.org/10.3389/fendo.2019.00931)

31 Shimon I, Adnan Z, Gorshtein A, Baraf L, Khazen NS, Gershinsky M, Pauker Y, Abid A, Niven MJ, Shechner C et al. Efficacy and safety of long-acting pasireotide in patients with somatostatin-resistant acromegaly: a multicenter study. Endocrine 201862 448-455. (https://doi.org/10.1007/s12020-018-1690-5)

32 Samson SL. Pasireotide in acromegaly: an overview of current mechanistic and clinical data. Neuroendocrinology 2015102 8-17. (https://doi.org/10.1159/000381460) 
33 Sheppard M, Bronstein MD, Freda P, Serri O, De Marinis L, Navers L, Rozhinskaya L, Resendiz KH, Ruffin M, Chen Y et al. Pasireotide LAR maintains inhibition of GH and IGF-1 in patients with acromegaly for up to 25 months: results from the blinded extension phase of a randomized, double-blind, multicenter, Phase III study. Pituitary 2015 18 385-394. (https://doi.org/10.1007/s11102-014-0585-6)

34 Bernabeu I, Rodriguez Gomez IA, Ramos-Levi AM \& Marazuela M. Profile of pegvisomant in the management of acromegaly: an evidence based review of its place in therapy. Research and Reports in Endocrine Disorders. Research and Reports in Endocrine Disorders 2015 5 47-58. (https://doi.org/10.2147/RRED.S78255)

35 Kasuki L, Wildemberg LEA, Neto LV, Marcondes J, Takiya CM \& Gadelha MR. Ki-67 is a predictor of acromegaly control with octreotide LAR independent of SST2 status and relates to cytokeratin pattern. European Journal of Endocrinology 2013169 217-223. (https:// doi.org/10.1530/EJE-13-0349)

36 Fusco A, Zatelli MC, Bianchi A, Cimino V, Tilaro L, Ventri F, Angelini F, Lauriola L, Vellone V, Doglietto F et al. Prognostic significance of the Ki-67 labeling index in growth hormone-secreting pituitary adenomas. Journal of Clinical Endocrinology and Metabolism 200893 2746-2750. (https://doi.org/10.1210/jc.2008-0126)

37 Gatto F, Feelders RA, Franck SE, Van Koetsveld PM, Dogan F, Kros JM, Neggers SJCMM, Van Der Lely AJ, Lamberts SWJ, Ferone D et al. In vitro head-to-head comparison between octreotide and pasireotide in GH-secreting pituitary adenomas. Journal of Clinical Endocrinology and Metabolism 2017102 2009-2018. (https://doi.org/10.1210/jc.2017. 00135)

38 Muhammad A, Coopmans EC, Gatto F, Franck SE, Janssen JAMJL, van der Lely AJ, Hofland LJ \& Neggers SJCMM. Pasireotide responsiveness in acromegaly is mainly driven by somatostatin receptor Subtype 2 expression. Journal of Clinical Endocrinology and Metabolism 2019104 915-924. (https://doi.org/10.1210/jc.2018-01524)

39 Marazuela M, Ramos-Leví AM, de Borges Souza P. \& Zatelli MC. Is receptor profiling useful for predicting pituitary therapy? European Journal of Endocrinology 2018179 D15-D25. (https://doi.org/10.1530/ EJE-18-0549)

40 Kasuki L, Wildemberg LE \& Gadelha MR. Management of endocrine disease: personalized medicine in the treatment of acromegaly. European Journal of Endocrinology 2018178 R89-R100. (https://doi. org/10.1530/EJE-17-1006)

41 Filopanti M, Giavoli C, Grottoli S, Bianchi A, De Marinis L, Ghigo E $\&$ Spada A. The exon 3-deleted growth hormone receptor: molecular and functional characterization and impact on GH/IGF-I axis in physiological and pathological conditions. Journal of Endocrinological Investigation 201134 861-868. (https://doi.org/10.1007/BF03346731)

42 Godowski PJ, Leung DW, Meacham LR, Galgani JP, Hellmiss R, Keret R, Rotwein PS, Parks JS, Laron Z \& Wood WI. Characterization of the human growth hormone receptor gene and demonstration of a partial gene deletion in two patients with Laron-type dwarfism. PNAS 198986 8083-8087. (https://doi.org/10.1073/pnas.86.20.8083)

43 Jorge AA, Marchisotti FG, Montenegro LR, Carvalho LR, Mendonca BB \& Arnhold IJP. Growth hormone (GH) pharmacogenetics: influence of GH receptor exon 3 retention or deletion on first-year growth response and final height in patients with severe GH deficiency. Journal of Clinical Endocrinology and Metabolism 200691 1076-1080. (https://doi.org/10.1210/jc.20052005)

44 Bianchi A, Giampietro A, Tartaglione L, Chiloiro S, Gentilella R, Bima C, Anile C, Olivi A, Pontecorvi A \& De Marinis L. Short- and long-term responsiveness to low dose growth hormone (GH) in adult GH deficiency: role of GH receptor polymorphism. Journal of Neuroendocrinology 201931 e12692. (https://doi.org/10.1111/ jne.12692)

45 Franck SE, Broer L, van der Lely AJ, Kamenicky P, Bernabeu I, Malchiodi E, Delhanty PJD, Rivadeneira F \& Neggers SJCMM. The effect of the exon-3-deleted growth hormone receptor on pegvisomant-treated acromegaly: a systematic review and metaanalysis. Neuroendocrinology 2017105 131-140. (https://doi. org/10.1159/000448844)

46 Chiloiro S, Mormando M, Bianchi A, Giampietro A, Milardi D, Bima C, Grande G, Formenti AM, Mazziotti G, Pontecorvi A et al. Prevalence of morphometric vertebral fractures in 'difficult' patients with acromegaly with different biochemical outcomes after multimodal treatment. Endocrine 201859 449-453. (https://doi. org/10.1007/s12020-017-1391-5)

47 Bianchi A, Mazziotti G, Tilaro L, Cimino V, Veltri F, Gaetani E, Pecorini G, Pontecorvi A, Giustina A \& De Marinis L. Growth hormone receptor polymorphism and the effects of pegvisomant in acromegaly. Pituitary 200912 196-199. (https://doi.org/10.1007/ s11102-008-0157-8)

48 Lasolle H, Ferriere A, Vasiljevic A, Eimer S, Nunes ML \& Tabarin A. Pasireotide-LAR in acromegaly patients treated with a combination therapy: a real-life study. Endocrine Connections 20198 1383-1394. (https://doi.org/10.1530/EC-19-0332)

Received 8 July 2020

Revised version received 14 October 2020

Accepted 2 November 2020 